\title{
Numerical representability of fuzzy total preorders
}

\author{
L. Agud ${ }^{1}$, R. G. Catalán ${ }^{2}$, S. Díaz ${ }^{3}$, E. Induráin*2 ${ }^{* 2}$ S. Montes ${ }^{3}$ \\ ${ }^{1}$ Department of Applied Mathematics, Polytechnic University of Valencia, Higher Polytechnic School of Alcoy, \\ Alcoy, Alicante 03801, Spain \\ lagudal@mat.upv.es \\ ${ }^{2}$ Department of Mathematics, Public University of Navarre, \\ Pamplona, Navarra 31006, Spain \\ raquel.garcia@unavarra.es, steiner@unavarra.es, \\ ${ }^{3}$ Department of Statistics and Operations Research, University of Oviedo, \\ Oviedo, Asturias 33071, Spain \\ diazsusana@uniovi.es,montes@uniovi.es \\ Received 20 March 2012 \\ Accepted 31 July 2012
}

\begin{abstract}
We introduce the concept of a fuzzy total preorder. Then we analyze its numerical representability through a real-valued order-preserving function defined for each $\alpha$-cut.
\end{abstract}

Keywords: Crisp and fuzzy binary relations, numerical representability, $\alpha$-cuts.

\section{Introduction}

Suppose that we consider different kinds of orderings on a set in order to establish some sort of comparison between its elements. When we formalize this setting, several classical kinds of binary relations (e.g. total preorders, interval orders, etc.) appear in a natural way. Usually, when dealing with these classes of relations, if we analyze if an element $a$ is related to another element $b$, it happens that the relationship is either void $(=0)$ or total $(=1)$ : Either the elements are not related at all, or they are fully related. No intermediate situation is allowed.
This is the crisp setting.

Nevertheless, a common feature arising in many models (e.g.: in Economics, Decision Making, etc.) is the consideration of comparisons or suitable binary relations -that constitute or represent the preferences of agents or decision-makers- that are graded. This is done in order to describe an intensity in the relationship between two given elements. In this case, two elements could be related at any level between 0 and 1 . Of course, now the binary relation becomes fuzzy.

Typical kinds of binary relations established for the crisp setting should be extended to the fuzzy set-

${ }^{*}$ Corresponding author: Esteban Induráin

Departamento de Matemáticas, Universidad Pública de Navarra. Campus Arrosadía, Edificio Las Encinas.

Pamplona, Navarra 31006, Spain.

Phone: +34-948169551; FAX: +34-948166057; e-mail: steiner@unavarra.es

Published by Atlantis Press

Copyright: the authors 
ting, in some appropriate way. However, it is indeed true that many equivalent definitions that appear in the crisp setting (e.g.: a total preorder, an interval order or a semiorder) fail to be equivalent when extended (in a natural way) to the fuzzy setting ${ }^{1,2,3,4}$.

In the crisp setting, classical studies on orderings deal with the key question of numerical representability. Roughly speaking, by means of some sort of numerical representation, if any, we translate the idea of comparing things or elements of a set through qualitative scales into the comparison of real numbers or quantitative scales. Thus, for instance, given a total preorder $\precsim$ defined on a nonempty set $X$ we may look for the existence of a real-valued function $u: X \rightarrow \mathbb{R}$ such that $a \precsim b \Leftrightarrow$ $u(a) \leqslant u(b)$ for every $a, b \in X$.

In the present paper we analyze this kind of questions for graded preferences, in the fuzzy setting. First we explain what we will understand for a representable fuzzy structure of a certain type. This discussion is important because, as mentioned before, a large variety of equivalent definitions of some kind of ordering in the crisp setting (e.g.: a crisp semiorder) could give rise to non-equivalent definitions in the fuzzy setting ${ }^{3,4}$.

As a matter of fact, in order to capture in a suitable way the idea of a representable fuzzy preference structure, a good election among those possible nonequivalent definitions of the concept to be analyzed is compulsory. In other words, not all the possible extended (non-equivalent) definitions of a concept in the fuzzy setting fit well with the analysis of numerical representability.

We establish the suitable definitions, that lean on the concept of representable $\alpha$-cut of a fuzzy binary relation. Then, the subsequent problem is the search for necessary and sufficient conditions that could characterize the existence of a numerical representation for the fuzzy preference structure.

In the present paper we analyze the numerical representability of fuzzy total preorders, extending some classical result that arises in the crisp setting.

The structure of the paper goes as follows:

After the Introduction, the notation and preliminaries are presented in Section 2. Preparatory results on $\alpha$-cuts are presented in Section 3. In Section 4 we introduce the key concept of a fuzzy total preorder. In Section 5 we analyze properties of representable additive fuzzy preference structures without incomparability. In Section 6 we study sufficient conditions for the representablity of a fuzzy total preorder. A Section 7 of further comments closes the paper.

\section{Notation and preliminaries}

\subsection{Basic concepts coming from the crisp setting}

When a crisp binary relation $\mathscr{Q}$ defined on a set $X$ connects two alternatives $a$ and $b$ we usually denote it by $a \mathscr{Q} b$. However, we can also consider a different notation. A crisp binary relation $\mathscr{Q}$ can be identified with a function $\mathscr{Q}: X \times X \rightarrow\{0,1\}$ that assigns the value 1 to the pair $(a, b)$ if and only if $a \mathscr{Q} b$ holds. Thus, it leads to the value 0 if and only if $\neg(a \mathscr{Q} b)$ holds. We will interchangeably use both notations for crisp relations along the paper, namely: $a \mathscr{Q} b$ or $\mathscr{Q}(a, b)=1$ will stand for $a$ being connected to $b$ by the relation $\mathscr{Q}$, whereas $\neg(a \mathscr{Q} b)$ or $\mathscr{Q}(a, b)=0$ will stand for $a$ not being connected to $b$ by $\mathscr{Q}$.

Definition 1. ${ }^{5}$ Let $X$ be a (nonempty) set and $\mathscr{P}$ be an asymmetric binary relation defined on $X$. Linked to $\mathscr{P}$, we define the binary relations $\mathscr{R}$ and $\mathscr{I}$ given by $a \mathscr{R} b \Longleftrightarrow \neg(b \mathscr{P} a)$, and $a \mathscr{I} b \Longleftrightarrow a \mathscr{R} b \wedge b \mathscr{R} a$ for every $a, b \in X$. The binary relation $\mathscr{R}$ (respectively, $\mathscr{I}$ ) is said to be the weak preference relation (respectively, the indifference relation) associated to $\mathscr{P}$.

(It is obvious that if $\mathscr{P}$ is asymmetric, then it is in particular irreflexive. Moreover, in this case $\mathscr{I}$ is reflexive.)

Given a nonempty set $X$ and an asymmetric binary relation $\mathscr{P}$ defined on $X$, the pair $(\mathscr{P}, \mathscr{I})$ is said to be a preference structure on $X$, the given set ${ }^{5}$.

(In the spirit of the ideas issued in a recent paper by Díaz, De Baets and Montes, we only deal with preference structures without incomparability. Otherwise the definition should be changed accordingly) ${ }^{2}$.

We immediately observe that, given a nonempty set $X$, a pair $(\mathscr{P}, \mathscr{I})$ of binary relations defined on $X$ is a preference structure if and only if it satisfies the following two conditions: 
(i) Given $a, b \in X$, exactly one of $a \mathscr{P} b, b \mathscr{P} a$, $a \mathscr{I} b$ obtains.

(ii) $a \mathscr{I} a$ for every $a \in X$.

(Notice that the fact of $\mathscr{P}$ being asymmetric directly follows from part (i) of this characterization.)

A crisp relation $\mathscr{Q}$ defined on a set $X$ is said to be total (also known as complete) if for every pair $a, b \in X$ it holds that $a \mathscr{Q} b$ or $b \mathscr{Q} a$.

Definition 2. ${ }^{5}$ A binary relation $\precsim$, defined on an arbitrary nonempty set $X$, is said to be a preorder if it is reflexive and transitive.

An antisymmetric preorder is said to be an order.

If a preorder $\precsim$ defined on $X$ is a total binary relation on $X$, then it is said to be a total preorder.

(Notice that this is equivalent to say that $\precsim$ is transitive and total, because, by definition, a total binary relation is in particular reflexive.)

If $\precsim$ is a preorder on $X$, then as usual we denote the associated asymmetric relation by $\prec$ and the associated equivalence relation by $\sim$ and these are defined, respectively, by $[a \prec b \Longleftrightarrow(a \precsim b) \wedge \neg(b \precsim$ $a)]$ and $[a \sim b \Longleftrightarrow(a \precsim b) \wedge(b \precsim a)]$. Also, the associated dual preorder $\precsim_{d}$ is defined by $\left[a \precsim_{d}\right.$ $b \Longleftrightarrow b \precsim a$.

Let $(X, \precsim)$ be a totally preordered set and let $X / \sim$ be the set of equivalence classes. If $a \in X$ we denote the equivalence class of $a$ by $[a]$. The preorder $\precsim$ on $X$ induces a natural linear order $\preceq$ on $X / \sim$ defined by $[a] \preceq[b] \Longleftrightarrow a \precsim b$.

Remark 1. In terms of Definition 1, and using the notation introduced there, it is straightforward to see that calling $\mathscr{P}=\prec$, it follows that $\mathscr{R}=\precsim$ and $\mathscr{I}=\sim$.

Definition 3. ${ }^{5}$ A total preorder $\precsim$ defined on a nonempty set $X$ is said to be representable if there exists a real-valued order-preserving map $u: X \rightarrow \mathbb{R}$ such that $a \precsim b \Longleftrightarrow u(a) \leqslant u(b)$, for every $a, b \in X$. The map $u$ is said to be a numerical representation (also known as utility function) for the total preorder $\precsim$.

\subsection{Fuzzy binary relations}

Definition 4. ${ }^{6}$ A fuzzy binary relation defined on a nonempty set $X$ is a function $\mathscr{Q}: X \times X \rightarrow[0,1]$.
Definition 5. ${ }^{7}$ A map $T:[0,1] \times[0,1] \rightarrow[0,1]$ is said to be a triangular norm (t-norm) if it satisfies the following conditions:

(i) Associativity: $T(T(x, y), z)=T(x, T(y, z))$ for every $x, y, z \in[0,1]$.

(ii) Commutativity: $T(x, y)=T(y, x)(x, y \in[0,1])$.

(iii) Monotonicity: $T(y, x) \leqslant T(z, x)$ for every $x, y, z \in$ $[0,1]$ such that $y \leqslant z$.

(iv) Boundary conditions: $T(x, 1)=x ; T(0,0)=$ $0(x \in[0,1])$.

Similarly, a map $S:[0,1] \times[0,1] \rightarrow[0,1]$ is said to be a triangular conorm (t-conorm) if it satisfies the conditions of associativity, commutativity, and monotonicity, as well as the following boundary conditions: $S(1,1)=1 ; S(x, 0)=x(x \in[0,1])$.

If $T$ is a $t$-norm, then the map $S:[0,1] \times[0,1] \rightarrow$ $[0,1]$ given by $S(x, y)=1-T(1-x, 1-y)$ for every $x, y \in[0,1]$ is a $t$-conorm, called dual or complementary of $T$ and it is usually denoted $S=T^{d}$. In the same way, if $S$ is a $t$-conorm then the map $T:[0,1] \times$ $[0,1] \rightarrow[0,1]$ defined by $T(x, y)=1-S(1-x, 1-y)$ for every $x, y \in[0,1]$ is a $t$-norm, called dual of $S$.

A $t$-norm $T$ is said to have zero-divisors if there exist two values $x, y \in(0,1)$ such that $T(x, y)=0$. (In this situation, $(x, y)$ is said to be a pair of zero divisors of the t-norm $T$ ).

It is necessary to recall that for fuzzy relations the definition of intersection and union are based on t-norms and t-conorms respectively.

Therefore also the composition of fuzzy relations depends on a t-norm $T$, so that given two fuzzy relations $\mathscr{Q}_{1}$ and $\mathscr{Q}_{2}$ defined on a set $X$, its composition as regards the t-norm $T$, denoted by $\mathscr{Q}_{1} \circ_{T} \mathscr{Q}_{2}$, is given by $\mathscr{Q}_{1} \circ_{T} \mathscr{Q}_{2}(a, b)=$ $\sup _{c \in X} T\left(\mathscr{Q}_{1}(a, c), \mathscr{Q}_{2}(c, b)\right) ; a, b \in X$.

There are multiple ways for defining the negation of a fuzzy relation. We consider the standard negation defined as follows: given a fuzzy binary relation $\mathscr{Q}$ on a nonempty set $X$, the negation $\mathscr{N} \mathscr{Q}$ of $\mathscr{Q}$ is given by $\mathscr{N} \mathscr{Q}(a, b)=1-\mathscr{Q}(a, b)$ for every $a, b \in X$.

Definition 6. ${ }^{6}$ For any t-norm $T$, a fuzzy relation $\mathscr{Q}$ is said to be:

(i) reflexive if $\mathscr{Q}(a, a)=1$, for every $a \in X$. 
(ii) $T$-asymmetric if $T(\mathscr{Q}(a, b), \mathscr{Q}(b, a))=0 ; a, b \in$ $X$.

(iii) $T$-transitive if $\mathscr{Q} \circ_{T} \mathscr{Q} \subseteq \mathscr{Q}$, that is, if $T(\mathscr{Q}(a, c), \mathscr{Q}(c, b)) \leqslant \mathscr{Q}(a, b)$, for every $a, b, c \in$ $X$,

(iv) $T$-negatively transitive if its negation $\mathscr{N} \mathscr{Q}$ is $T$ transitive.

Let us observe that if a t-norm $T$ has no pair of zero-divisors, then a $T$-asymmetric fuzzy relation $\mathscr{Q}$ may satisfy that there exist two alternatives $a$ and $b \in X$ such that $\min (\mathscr{Q}(a, b), \mathscr{Q}(b, a))>0$.

Notice also that a fuzzy relation $\mathscr{Q}$ cannot be simultaneously reflexive and $T$-asymmetric, since the fact $\mathscr{Q}(a, a)=1$ is incompatible with $T(\mathscr{Q}(a, a), \mathscr{Q}(a, a))=0$. (Indeed, $T(1,1)=1$ because $T$ is a t-norm).

Definition 7. ${ }^{6}$ For any t-conorm $S$, a fuzzy relation $\mathscr{Q}$ defined on a nonempty set $X$ is called $S$-complete if $S(\mathscr{Q}(a, b), \mathscr{Q}(b, a))=1$ for every $a, b \in X$. The two most important notions of $S$-completeness are the strong completeness (when $S=S_{M}$ is the maximum t-conorm defined by $S_{M}(x, y)=\max \{x, y\}$ for every $x, y \in[0,1])$, and the weak completeness (when $S=S_{L}$ is the Łukasiewicz t-conorm defined by $S_{L}(x, y)=\min \{1, x+y\}$ for every $\left.x, y \in[0,1]\right)$.

(Further information about the concept of $S$ completeness in the fuzzy setting may be seen in the paper by I. Montes et al. mentioned in the references) ${ }^{1}$.

\subsection{Additive fuzzy preference structures}

Several authors have debated about the notion of preference structure in the setting of fuzzy relations until Van de Walle et al. introduced the notion of an additive fuzzy preference structure.

Definition 8. ${ }^{8,9}$ An additive fuzzy preference structure on a nonempty set $X$ is a triplet of fuzzy binary relations $(\mathscr{P}, \mathscr{I}, \mathscr{J})$ satisfying that $\mathscr{I}$ is reflexive and symmetric $(\mathscr{I}(a, a)=1 ; \mathscr{I}(a, b)=\mathscr{I}(b, a)$ for every $a, b \in X)$ and $\mathscr{P}(a, b)+\mathscr{P}^{t}(a, b)+\mathscr{I}(a, b)+$ $\mathscr{J}(a, b)=1$ for any $a, b \in X$, where $\mathscr{P}^{t}$ denotes the transpose (or converse) of $\mathscr{P}\left(\mathscr{P}^{t}(a, b)=\right.$ $\mathscr{P}(b, a) ; \quad a, b \in X)$ and $\mathscr{J}$ denotes the binary relation of incomparability.
Throughout this paper, we will only deal with additive fuzzy preference structures without incomparability (i.e. $\mathscr{J}(a, b)=0$ for any $a, b \in X$, so that accordingly we use the notation $(\mathscr{P}, \mathscr{I})$ to deal with an additive fuzzy preference structure without incomparability). These fuzzy preference structures have a very important property (that remind us Definition 1 given in the crisp setting). Namely, in an additive fuzzy preference structure without incomparability the relation $\mathscr{P}$ immediately determines the relation $\mathscr{I}$. As a matter of fact, for any pair of alternatives $a, b \in X$ we have that:

$$
\begin{aligned}
\mathscr{I}(a, b) & =1-\mathscr{P}(a, b)-\mathscr{P}^{t}(a, b) \\
& =1-\mathscr{P}(a, b)-\mathscr{P}(b, a) .
\end{aligned}
$$

The relation $\mathscr{P}$ is said to be a fuzzy strict preference relation and it shows the degree with which the decision maker strictly prefers $a$ to $b$. The relation $\mathscr{I}$ is called the fuzzy indifference relation and shows the level with which $a$ and $b$ are considered indifferent.

Every additive fuzzy preference structure has associated a fuzzy weak preference relation $\mathscr{R}$ given by $\mathscr{R}(a, b)=\mathscr{P}(a, b)+\mathscr{I}(a, b)$ for every $a, b \in X$. The value $\mathscr{R}(a, b)$ expresses the degree of truth of the assertion " $a$ is at least as good as b".

Observe that if we are dealing with an additive fuzzy preference structure without incomparability, then we can recover $\mathscr{P}$ and $\mathscr{I}$ from $\mathscr{R}$ as follows: $\mathscr{P}(a, b)=1-\mathscr{R}(b, a) ; \mathscr{I}(a, b)=\mathscr{R}(a, b)+$ $\mathscr{R}(b, a)-1$, for every $a, b \in X$. Observe also that $\mathscr{R}(a, a)=\mathscr{P}(a, a)+\mathscr{I}(a, a)=0+1=1$, for every $a \in X$.

Remark 2. Needless to say that other possible definitions of the notions of a fuzzy preference, or of a fuzzy utility function, may undoubtedly deserve interest ${ }^{10,11,12,13,14,15,16,17,18,19,20}$. A comparison between those settings and the one we follow here, namely the approach introduced by Van de Walle et al., could be appealing and illuminate the panorama. However, in our opinion, this could be material for new pieces of research, because of the wide sort of variations and nuances in the corresponding key concepts, whose study in depth, if made in the present paper, would unnecessarily en- 
large the manuscript, perhaps concealing the main ideas we have in mind.

To summarize, we will follow the approach that, we think, fits better the highlight intended for this article, namely the analysis of the problem of a numerical representation in the fuzzy setting in terms of $\alpha$-cuts.

\section{The $\alpha$-cuts of a fuzzy binary relation}

The results of this preparatory Section 3 deal with compatibility between additive fuzzy preference structures without incomparability and their $\alpha$-cuts. Proposition 3 has already been stated and proved in the subsection 4.1 of the paper by E. Induráin et al. mentioned in the references ${ }^{4}$. Proposition 4 follows directly from definitions. Similar results appear also in a recent paper by S. Díaz, B. De Baets and S. Montes $^{21}$.

Definition 9. Given a fuzzy relation $\mathscr{Q}$ on a nonempty set $X$, and a real number $\alpha \in[0,1]$, we define the $\alpha$-cut of $\mathscr{Q}$, denoted $\mathscr{Q}_{\alpha}$, as the crisp binary relation on $X$ given by $\mathscr{Q}_{\alpha}=\left\{(a, b) \in X^{2}\right.$ : $\mathscr{Q}(a, b) \geqslant \alpha\}$.

The set of all the fuzzy binary relations on $X$ is denoted $\mathscr{Q}_{X}$.

A fuzzy binary relation $\mathscr{Q}$ defined on a nonempty set $X$, can be identified with the family of its $\alpha$-cuts, that are crisp binary relations. Actually, a nested family of (crisp) binary relations, indexed in $[0,1]$, may also be identified to a fuzzy binary relation, in a way that each element of the family corresponds to an $\alpha$-cut. Let us formalize all this through the following Definition 10 and Proposition 1.

Definition 10. A family $\left\{\mathscr{Q}_{\alpha}: \alpha \in[0,1]\right\}$ of crisp binary relations defined on a nonempty set $X$ is said to be fuzzy generating if it satisfies the following two conditions:

(i) For every $0 \leqslant \beta \leqslant \alpha \leqslant 1, G\left(\mathscr{Q}_{\alpha}\right)=\{(a, b) \in X \times$ $\left.X: \mathscr{Q}_{\alpha}(a, b)=1\right\} \subseteq G\left(\mathscr{Q}_{\beta}\right)=\{(a, b) \in X \times X:$ $\left.\mathscr{Q}_{\beta}(a, b)=1\right\}$.

(ii) For every $(a, b) \in X \times X$ there exists $\alpha \in[0,1]$ with $\mathscr{Q}_{\alpha}(a, b)=1$, and satisfying also that $\alpha<$ $1 \Rightarrow \mathscr{Q}_{\beta}(a, b)=0$, for every $\beta>\alpha$.
The set of fuzzy generating families on $X$ is denoted $\mathscr{F}_{X}$.

The next straightforward Proposition 1 establishes the relationship between $\mathscr{Q}_{X}$ and $\mathscr{F}_{X}$ : They can be identified, so that a fuzzy generating family can be interpreted, directly, as the collection of $\alpha$ cuts of a fuzzy binary relation.

Proposition 1. Let $X$ be a nonempty set. The following assertions hold true:

(a) The map $\gamma: \mathscr{Q}_{X} \rightarrow \mathscr{F}_{X}$ given by $\gamma(\mathscr{Q})=\left\{\mathscr{Q}_{\alpha}\right.$ : $\alpha \in[0,1]\}$ is well-defined and injective.

(b) The map $\mu: \mathscr{F}_{X} \rightarrow \mathscr{Q}_{X}$ given by $\mu\left(\left\{\mathscr{Q}_{\alpha}\right.\right.$ : $\alpha \in[0,1]\})(a, b)=\max \left\{\alpha \in[0,1]: \mathscr{Q}_{\alpha}(a, b)=\right.$ 1\} $(a, b \in X)$ is well-defined and injective.

(c) $\mu=\gamma^{-1}$.

Therefore $\mathscr{Q}_{X}$ can be identified with $\mathscr{F}_{X}$ in a natural way.

\section{Proof.}

(a) The map $\gamma$ is well-defined since for any fuzzy relation $\mathscr{Q}$, the collection $\left\{\mathscr{Q}_{\alpha}: \alpha \in[0,1]\right\}$ is a fuzzy generating family:

(i) For all $0 \leqslant \beta \leqslant \alpha \leqslant 1, \mathscr{Q}_{\alpha}(a, b)=1$ implies $\mathscr{Q}(a, b) \geqslant \alpha \geqslant \beta$. And this implies $\mathscr{Q}_{\beta}(a, b)=$ 1 , so $G\left(\mathscr{Q}_{\alpha}\right) \subseteq G\left(\mathscr{Q}_{\beta}\right)$.

(ii) Obviously, for every $(a, b) \in X$, calling $\alpha=$ $\mathscr{Q}(a, b)$ it holds that if $\alpha<1$ then $\mathscr{Q}_{\beta}(a, b)=0$ for every $\beta>\alpha$.

(This follows from the definition of the $\alpha$-cuts of the fuzzy binary relation $\mathscr{Q}$ ).

On the other hand, the map $\gamma$ is injective. If we consider two fuzzy relations $\mathscr{Q}$ and $\mathscr{R}$ defined on $X$ such that they are different, i.e., such that there are two alternatives $a, b \in X$ satisfying that $\mathscr{Q}(a, b) \neq \mathscr{R}(a, b)$, then $\gamma(\mathscr{Q}) \neq \gamma(\mathscr{R})$ :

Assume without loss of generality that $\alpha=$ $\mathscr{Q}(a, b)<\mathscr{R}(a, b)=\beta$, then $\mathscr{R}_{\beta}(a, b)=1$ but $\mathscr{Q}_{\beta}(a, b)=0$, so $\mathscr{R}_{\beta} \neq \mathscr{Q}_{\beta}$ and therefore $\gamma(\mathscr{Q}) \neq$ $\gamma(\mathscr{R})$.

(b) First of all observe that for all $\left\{\mathscr{Q}_{\alpha}: \alpha \in[0,1]\right\} \in$ $\mathscr{F}_{X}$ and for all $a, b \in[0,1]$, the set $\{\alpha \in[0,1]$ : $\left.\mathscr{Q}_{\alpha}(a, b)=1\right\}$ admits a maximum. This follows from part (ii) of Definition 10. Then the relation $\mathscr{Q}$ defined on $X \times X$ such that for all $a, b \in X$ takes 
the value $\max \left\{\mathscr{Q}_{\alpha}: \alpha \in[0,1]\right\} \in \mathscr{F}_{X}$ is welldefined since it takes values on $[0,1]$.

The map $\mu$ is injective. If we consider two different sets $\left\{\mathscr{Q}_{\alpha}: \alpha \in[0,1]\right\},\left\{\mathscr{R}_{\alpha}: \alpha \in[0,1]\right\} \in \mathscr{F}_{X}$, then there is a value $\alpha \in[0,1]$ such that $\mathscr{Q}_{\alpha} \neq \mathscr{R}_{\alpha}$, that is, there are two alternatives $a, b \in X$ such that $\mathscr{Q}_{\alpha}(a, b) \neq \mathscr{R}_{\alpha}(a, b)$. Assume now without loss of generality that $\mathscr{Q}_{\alpha}(a, b)=0 \neq 1=$ $\mathscr{R}_{\alpha}(a, b)$. In this case, by Definition 10 (i), we have that $\mathscr{Q}_{\beta}(a, b)=0$ for all $\beta>\alpha$. Hence $\mu\left(\left\{\mathscr{Q}_{\alpha}: \alpha \in[0,1]\right\}\right)(a, b)<\alpha$, whereas $\mu\left(\left\{\mathscr{R}_{\alpha}\right.\right.$ : $\alpha \in[0,1]\})(a, b) \geqslant \alpha$, so that the images are actually different.

(c) This follows from the construction of both functions $\gamma$ and $\mu$.

Remark 3. When considering an additive fuzzy preference structure without incomparability $(\mathscr{P}, \mathscr{I})$, whose associated fuzzy weak preference relation is $\mathscr{R}$, defined on a nonempty set $X$, the pair $\left(\mathscr{P}_{\alpha}, \mathscr{I}_{\alpha}\right)$ may fail to be a crisp preference structure. To see this, consider the example $X=$ $\{a, b\}$, endowed with the additive fuzzy preference structure $(\mathscr{P}, \mathscr{I})$ given by $\mathscr{P}(a, a)=\mathscr{P}(b, b)=$ $0 ; \mathscr{I}(a, a)=\mathscr{I}(b, b)=1 ; \mathscr{P}(a, b)=\mathscr{P}(b, a)=$ $\mathscr{I}(a, b)=\mathscr{I}(b, a)=\frac{1}{3}$. In this case, for $\alpha=\frac{1}{2}$ it is plain that $\mathscr{P}_{\alpha}(a, b)=\mathscr{P}_{\alpha}(b, a)=\mathscr{I}_{\alpha}(a, b)=0$, so that $\left(\mathscr{P}_{\alpha}, \mathscr{I}_{\alpha}\right)$ is not a preference structure in the sense of Definition 1.

As a matter of fact, the situation shown in Remark 3 is the expected one when dealing with additive fuzzy preference structures without incomparability. The only exception occurs when $(\mathscr{P}, \mathscr{I})$ is indeed a crisp preference structure.

Theorem 2. ${ }^{4}$ An additive fuzzy preference structure without incomparability $(\mathscr{P}, \mathscr{I})$, defined on a nonempty set $X$, satisfies that all its $\alpha$-cuts for $\alpha>0$ are crisp preference structures if and only if it is a crisp preference structure.

Due to the fact stated in Remark 3 and Theorem 2 , in what follows we systematically consider as $\alpha$ cut of an additive fuzzy preference structure without incomparability $(\mathscr{P}, \mathscr{I})$, defined on a nonempty set $X$, the $\alpha$-cut (namely, $\mathscr{R}_{\alpha}$ ) of the fuzzy weak preference relation $\mathscr{R}$ corresponding to $(\mathscr{P}, \mathscr{I})$.
With respect to the relationship between the $\alpha$ cuts $\mathscr{R}_{\alpha}$ and the behavior of the the fuzzy weak preference relation $\mathscr{R}$, the following results complete the panorama.

Proposition 3. ${ }^{4}$ Let $(\mathscr{P}, \mathscr{I})$ be an additive fuzzy preference structure without incomparability, defined on a nonempty set $X$. The following statements are equivalent:

(a) The $\alpha$-cut $\mathscr{R}_{\alpha}$ is total for every $\alpha \in[0,1]$.

(b) $\min \{\mathscr{P}(a, b), \mathscr{P}(b, a)\}=0$ for every $a, b \in X$.

(c) $\mathscr{R}$ is strongly complete.

Proposition 4. Let $(\mathscr{P}, \mathscr{I})$ be an additive fuzzy preference structure without incomparability, defined on a nonempty set $X$. The following statements are equivalent:

(a) The $\alpha$-cut $\mathscr{R}_{\alpha}$ is transitive for every $\alpha \in[0,1]$.

(b) $\mathscr{R}$ is $T_{M}$-transitive, where $T_{M}$ stands for the minimum t-norm.

Proof. (a) $\Rightarrow$ (b) For any three alternatives $a, b, c \in$ $X$, let $\alpha=\min \{\mathscr{R}(a, b), \mathscr{R}(b, c)\}$. It follows that $\mathscr{R}_{\alpha}(a, b)=1=\mathscr{R}_{\alpha}(b, c)$. Moreover, since $\mathscr{R}_{\alpha}$ is transitive we get $\mathscr{R}_{\alpha}(a, c)=1$. This implies that $\mathscr{R}(a, c) \geqslant \alpha=\min \{\mathscr{R}(a, b), \mathscr{R}(b, c)\}$.

(b) $\Rightarrow$ (a) Fix a value $\alpha \in[0,1]$. If $\mathscr{R}_{\alpha}(a, b)=1$ and $\mathscr{R}_{\alpha}(b, c)=1$, then $\mathscr{R}(a, b) \geqslant \alpha$ and $\mathscr{R}(b, c) \geqslant$ $\alpha$. Hence $\mathscr{R}(a, c) \geqslant \min \{\mathscr{R}(a, b), \mathscr{R}(b, c)\} \geqslant \alpha$. Therefore $\mathscr{R}_{\alpha}(a, c)=1$, so that $\mathscr{R}_{\alpha}$ is transitive.

\section{Fuzzy total preorders}

When dealing with total preorders in the crisp setting, it is well known that if $\precsim$ is a total preorder defined on a nonempty set $X$ then its associated binary relation $\prec$ is asymmetric and negatively transitive. The converse is also true: If $\prec$ is asymmetric and negatively transitive, and we define $\precsim$ as $a \precsim b \Leftrightarrow \neg(b \prec a)$ for every $a, b \in X$, then $\precsim$ is actually a total preorder on $X$.

Moreover, the existence of a utility function $u$ : $X \rightarrow \mathbb{R}$ representing $\precsim$ is equivalent to the existence of a real-valued map $v: X \rightarrow \mathbb{R}$ such that $a \prec b \Leftrightarrow v(a)<v(b)$ for every $a, b \in X$. (Indeed, we may take $u=v$ ). In other words, the representability 
of $\precsim$ immediately carries a "representability" of its corresponding asymmetric part $\prec$, and viceversa.

Let us see what happens when these ideas are extended to the fuzzy setting.

Definition 11. ${ }^{6}$ Let $(\mathscr{P}, \mathscr{I})$ be an additive fuzzy preference structure without incomparability, defined on a nonempty set $X$. Let $T$ be a $t$-norm and $S=T^{d}$ its dual $t$-conorm. The structure $(\mathscr{P}, \mathscr{I})$ is said to be a fuzzy total T-preorder if the corresponding fuzzy relation $\mathscr{R}$, given by $\mathscr{R}(a, b)=$ $\mathscr{P}(a, b)+\mathscr{I}(a, b)$ for every $a, b \in X$, is reflexive, $T$-transitive and $S$-complete.

Given an additive fuzzy preference structure without incomparability $(\mathscr{P}, \mathscr{I})$, defined on a nonempty set $X$, it is clear that the $\alpha$-cut $\mathscr{R}_{\alpha}$, of the associated fuzzy weak preference relation $\mathscr{R}$, is reflexive for every $\alpha \in[0,1]$. So, the following result is now a direct consequence of Proposition 3 and Proposition 4.

Corollary 5. Let $(\mathscr{P}, \mathscr{I})$ be an additive fuzzy preference structure without incomparability, defined on a nonempty set $X$, and $\mathscr{R}$ its associated fuzzy weak preference relation. Let $T_{M}$ be the minimum $t$-norm. The following assertions are equivalent:

(a) The $\alpha$-cut $\mathscr{R}_{\alpha}$ is a total preorder, for every $\alpha \in$ $[0,1]$.

(b) The structure $(\mathscr{P}, \mathscr{I})$ is a fuzzy total $T_{M^{-}}$ preorder.

Through the following Proposition 6, in its subsequent Corollary 7 we obtain a characterization of the concept of a fuzzy total preorder.

Proposition 6. Let $T$ be a $t$-norm and $S=T^{d}$ its dual $t$-conorm. Let $(\mathscr{P}, \mathscr{I})$ be an additive fuzzy preference structure without incomparability, defined on a nonempty set $X$. The following statements hold:

(a) $\mathscr{R}$ is $S$-complete if and only if $\mathscr{P}$ is $T$ asymmetric.

(b) $\mathscr{R}$ is $T$-transitive if and only if $\mathscr{P}$ is $T$-negatively transitive.

Proof. (a) (Notice that this generalizes the equivalence (b) $\Leftrightarrow$ (c) of Proposition 3, since in Proposition 3 the equivalence only concerns the minimum $\mathrm{t}$-norm and in this item the equivalence is assured for any t-norm $T$ ). For every $a, b \in X$ we have that $\mathscr{R}$ is $S$-complete $\Leftrightarrow S(\mathscr{R}(a, b), \mathscr{R}(b, a))=1 \Leftrightarrow 1-T(1-$ $\mathscr{R}(a, b), 1-\mathscr{R}(b, a))=1 \Leftrightarrow T(1-\mathscr{R}(a, b), 1-$ $\mathscr{R}(b, a))=0 . \Leftrightarrow T(\mathscr{P}(b, a), \mathscr{P}(a, b))=0 \Leftrightarrow \mathscr{P}$ is $T$-asymmetric. (Observe here that $(\mathscr{P}, \mathscr{I})$ is an additive fuzzy preference structure without incomparability, so that $\mathscr{P}(a, b)+\mathscr{P}(b, a)+\mathscr{I}(a, b)=1=$ $\mathscr{P}(b, a)+\mathscr{R}(a, b)$. In other words, $1-\mathscr{R}(a, b)=$ $\mathscr{P}(b, a)$ for every $a, b \in X)$.

(b) This part has already been proved in the literature: see Theorem 1 in the paper by S. Díaz et al. (2012) mentioned in the references ${ }^{21}$.

Remark 4. Part $\mathrm{b}$ of Proposition 6 has not an analogue for $\alpha$-cuts. That is, if $(\mathscr{P}, \mathscr{I})$ is an additive fuzzy preference structure without incomparability, defined on a nonempty set $X$, and its fuzzy weak preference relation is $\mathscr{R}$, for some $\alpha \in[0,1]$ it may happen that $\mathscr{R}_{\alpha}$ is transitive but $\mathscr{P}_{\alpha}$ fails to be negatively transitive. (See Example 2 in next section). The reason is that given a fuzzy binary relation $\mathscr{Q}$ on a nonempty set $X$, and fixed $\alpha \in[0,1]$, it is not true, in general, that the $\alpha$-cut $(\mathscr{N} \mathscr{Q})_{\alpha}$ of the negation of $\mathscr{Q}$ coincides with the (crisp) negation $\neg\left(Q_{\alpha}\right)$ of the $\alpha$-cut $\mathscr{Q}_{\alpha}$.

Corollary 7. Let $T$ be a t-norm and $S=T^{d}$ its dual $t$-conorm. An additive fuzzy preference structure without incomparability $(\mathscr{P}, \mathscr{I})$, defined on a nonempty set $X$, is a fuzzy total $T$-preorder if and only if $\mathscr{P}$ is $T$-asymmetric and $T$-negatively transitive.

Proof. Notice that since $\mathscr{R}$ is obtained from the given additive fuzzy preference structure without incomparability, as $\mathscr{R}(a, b)=\mathscr{P}(a, b)+\mathscr{I}(a, b)$, it follows that $\mathscr{P}$ is already reflexive, since by Definition $8, \mathscr{I}$ is reflexive. Thus, to conclude it is enough to prove that $\mathscr{R}$ is $T$-transitive and $S$-complete if and only if $\mathscr{P}$ is $T$-asymmetric and $T$-negatively transitive. But this follows from Proposition 6.

Corollary 8. If an additive fuzzy preference structure without incomparability $(\mathscr{P}, \mathscr{I})$, defined on a nonempty set $X$, is a fuzzy total $T_{M}$-preorder, then it 
is also a fuzzy total T-preorder for any t-norm $T$.

Proof. Notice that $T_{M}$ is the biggest t-norm, so that if $\mathscr{P}$ is $T_{M}$-asymmetric and $T_{M}$-negatively transitive, it is also $T$-asymmetric and $T$-negatively transitive for any t-norm $T$, by definition. Then use Corollary 7 to conclude.

The converse of Corollary 8 is not true as can be seen in the next Example 1:

Example 1. Consider the following additive fuzzy preference structure without incomparability $(\mathscr{P}, \mathscr{I})$, with associated fuzzy weak preference relation $\mathscr{R}$, defined on the set $X=\{a, b\}$ as follows:

\begin{tabular}{c|cccc|cc}
$\mathscr{P}$ & $a$ & $b$ & & $\mathscr{I}$ & $a$ & $b$ \\
\hline$a$ & 0 & 0.5 & & $a$ & 1 & 0 \\
$b$ & 0.5 & 0 & & $b$ & 0 & 1 \\
\multicolumn{4}{c}{} \\
& $\mathscr{R}$ & $a$ & $b$ & & \\
\cline { 2 - 3 } & $a$ & 1 & 0.5 & & \\
& $b$ & 0.5 & 1 &
\end{tabular}

An easy checking shows that $(\mathscr{P}, \mathscr{I})$ is a fuzzy total $T_{L}$-preorder with respect to the Łukasiewicz tnorm given by $T_{L}(x, y)=\max \{0, x+y-1\}(x, y \in$ $[0,1])$. However it is not a fuzzy total $T_{M^{-}}$ preorder with respect to the minimum t-norm, since $\min \{\mathscr{P}(a, b), \mathscr{P}(b, a)\}=0.5 \neq 0$, so that $\mathscr{P}$ is not $T_{M}$-asymmetric.

\section{Representability of additive fuzzy preference structures without incomparability}

In the present section we introduce the concepts of representability and quasi-representability of additive fuzzy preference structures without incomparability (see Definition 12 below). In our approach, we will deal with the representability of an additive fuzzy preference structure $(\mathscr{P}, \mathscr{I})$ whose fuzzy weak preference relation is $\mathscr{R}$, in terms of representability of the corresponding $\alpha$-cuts $\mathscr{P}_{\alpha}$ (respectively, $\mathscr{R}_{\alpha}$ ) as crisp binary relations that are asymmetric (hence irreflexive, in particular) and negatively transitive (respectively, that are total preorders). The representations considered use a single real-valued utility function $u_{\alpha}$. These ideas could be extended to study another kinds of representability of additive fuzzy preference structures $(\mathscr{P}, \mathscr{I})$ in terms of the representability of its $\alpha$-cuts $\mathscr{R}_{\alpha}$ by means of two real-valued functions $u_{\alpha}, v_{\alpha}$ such that $x \mathscr{R}_{\alpha} y \Leftrightarrow u_{\alpha}(a) \leqslant v_{\alpha}(b)$, for every $a, b \in X$ (as interval orders). We may also consider representations by means of a function $u_{\alpha}$ and a positive threshold of discrimination $k>0$ such that $a \mathscr{R}_{\alpha} b \Leftrightarrow u_{\alpha}(a) \leqslant$ $u_{\alpha}(b)+k$, for every $a, b \in X$ (now as semiorders, in the sense of Scott and Suppes) $)^{22,23,24,4}$.

These new possibilities that we point out here are left for future research: in the present paper we will study only the case in which the representations of the $\alpha$-cuts use a single real valued function. We will be working mainly with the $\alpha$-cuts $\mathscr{R}_{\alpha}$, but providing also some result on the representability of the $\alpha$-cuts $\mathscr{P}_{\alpha}$. This will allow us to understand better the concept of a fuzzy total preorder, through its representability properties.

Definition 12. Let $T$ be a $t$-norm and $S=T^{d}$ its dual $t$-conorm. An additive fuzzy preference structure without incomparability $(\mathscr{P}, \mathscr{I})$, defined on a nonempty set $X$, is said to be:

(i) representable if for every $\alpha \in[0,1]$ the $\alpha$-cut $\mathscr{R}_{\alpha}$ of the associated fuzzy binary relation $\mathscr{R}$ is a representable (crisp) total preorder, in the sense of Definition 3.

(ii) quasi-representable if for every $\alpha \in(0,1]$ the $\alpha$ cut $\mathscr{P}_{\alpha}$ of the fuzzy relation $\mathscr{P}$ satisfies that there exists a real-valued map $u_{\alpha}: X \rightarrow \mathbb{R}$ such that $a \mathscr{P}_{\alpha} b \Leftrightarrow u_{\alpha}(a)<u_{\alpha}(b)$ for every $a, b \in X$.

Remark 5. Notice that, in particular, if $(\mathscr{P}, \mathscr{I})$ is quasi-representable, then the $\alpha$-cut $\mathscr{P}_{\alpha}$ of the fuzzy relation $\mathscr{P}$ is a (crisp) negatively transitive relation, for every $\alpha \in(0,1]$. The converse is not true in general: there exist crisp total preorders whose asymmetric part, that is negatively transitive, can not be represented in the real line through a utility function. An example is the asymmetric part of the lexicographic order defined on the real plane ${ }^{25,26}$.

Observe also that, in the definition of quasirepresentability, we always take $\alpha \neq 0$. The reason is that, by definition, $\mathscr{P}_{0}(a, b)=1$ for every $a, b \in X$, so that the existence of the corresponding 
real-valued map $u_{0}$ for $\mathscr{P}_{0}$ would immediately lead to the contradiction $u_{0}(a)<u_{0}(b)<u_{0}(a)$.

Finally, observe that if $(\mathscr{P}, \mathscr{I})$ is representable by means of a family of real-valued functions $\left\{u_{\alpha}\right\}_{\alpha \in[0,1]}$ such that $a \mathscr{R}_{\alpha} b \Leftrightarrow u_{\alpha}(a) \leqslant u_{\alpha}(b)(a, b \in$ $X)$, then, for any $a, b \in X$, it holds that $\mathscr{R}(a, b)=$ $\beta \Rightarrow u_{\alpha}(a) \leqslant u_{\alpha}(b)$ for every $\alpha \leqslant \beta$ and also $u_{\alpha}(a)>u_{\alpha}(b)$ for every $\alpha>\beta$.

In the same way, if $(\mathscr{P}, \mathscr{I})$ is quasirepresentable by means of a family of real-valued functions $\left\{v_{\alpha}\right\}_{\alpha \in(0,1]}$ such that $a \mathscr{P}_{\alpha} b \Leftrightarrow v_{\alpha}(a)<$ $v_{\alpha}(b)(a, b \in X)$, then, for any $a, b \in X$, it holds that $\mathscr{P}(a, b)=\beta \Rightarrow v_{\alpha}(a)<v_{\alpha}(b)$ for every $\alpha \leqslant \beta$ and also $v_{\alpha}(a) \geqslant v_{\alpha}(b)$ for every $\alpha>\beta$.

Remark 6. By the equivalence (a) $\Leftrightarrow$ (c) in Proposition 3, a necessary condition for an additive fuzzy preference structure without incomparability $(\mathscr{P}, \mathscr{I})$, defined on a nonempty set $X$, to be representable is the strong completeness (i.e.: $S_{M^{-}}$ completeness, where $S_{M}$ stands for the maximum $t$ conorm) of the associated fuzzy relation $\mathscr{R}$. (Observe that indeed this does not depend on the t-norm $T$ considered).

By Theorem 2, given an $\alpha$-cut, in general $\left\{\mathscr{P}_{\alpha}, \mathscr{I}_{\alpha}\right\}$ fails to be a (crisp) preference structure, so that, in particular $\mathscr{P}_{\alpha}$ will not coincide with the negation of the transpose of $\mathscr{R}_{\alpha}$, as next Example 2 shows.

The existence of a real-valued map $u_{\alpha}: X \rightarrow \mathbb{R}$ such that $a \mathscr{R}_{\alpha} b \Longleftrightarrow u_{\alpha}(a) \leqslant u_{\alpha}(b)+1$, for every $a, b \in X$, immediately forces the crisp binary relation $\mathscr{R}_{\alpha}$ to be total. As a matter of fact, for every $a, b \in X$ we have that if $a \mathscr{R}_{\alpha} b$ does not hold, then $u_{\alpha}(b)+1<u_{\alpha}(a)$. Hence $u_{\alpha}(b)<u_{\alpha}(a)-1<$ $u_{\alpha}(a)+1$, so that $b \mathscr{R}_{\alpha} a$ holds. Anyways, as a consequence of Proposition 3, we already knew that the $\alpha$-cuts $\mathscr{R}_{\alpha}$ are total whenever $(\mathscr{P}, \mathscr{I})$ is a fuzzy total $T$-preorder, for some t-norm $T$.

When $\mathscr{R}_{\alpha}$ is a total binary relation, if we define new binary relations $\mathscr{S}_{\alpha}, \mathscr{T}_{\alpha}$ on $X$ by $a \mathscr{S}_{\alpha} b \Longleftrightarrow$ $\neg\left(b \mathscr{R}_{\alpha} a\right)$, and $a \mathscr{T}_{\alpha} b \Longleftrightarrow\left(a \mathscr{R}_{\alpha} b \wedge b \mathscr{R}_{\alpha} a\right)$, for every $a, b \in X$, it is straightforward to see that $\mathscr{S}_{\alpha}$ is asymmetric, and $\left(\mathscr{S}_{\alpha}, \mathscr{T}_{\alpha}\right)$ is a crisp preference structure whose associated weak preference relation is $\mathscr{R}_{\alpha}$. Finally, observe that $\left(\mathscr{S}_{\alpha}, \mathscr{T}_{\alpha}\right)$ has nothing to $d o$, in general, with the ordered pair $\left(\mathscr{P}_{\alpha}, \mathscr{I}_{\alpha}\right)$ that comes from the given additive fuzzy preference structure $(\mathscr{P}, \mathscr{I})$ after taking its $\alpha$-cut. Indeed, by Theorem 2, $\left(\mathscr{S}_{\alpha}, \mathscr{T}_{\alpha}\right)$ and $\left(\mathscr{P}_{\alpha}, \mathscr{I}_{\alpha}\right)$ only coincide when the given preference structure $(\mathscr{P}, \mathscr{I})$ is crisp. (Example 2 below also shows that they are different in general: consider e.g. the 0.75 -cut).

Example 2. Consider the following additive fuzzy preference structure without incomparability $(\mathscr{P}, \mathscr{I})$, with associated fuzzy weak preference relation $\mathscr{R}$, defined on the set $X=\{a, b, c, d\}$ as follows:

\begin{tabular}{c|cccc}
$\mathscr{P}$ & $a$ & $b$ & $c$ & $d$ \\
\hline$a$ & 0 & 0 & 0.8 & 0.7 \\
$b$ & 0 & 0 & 0.3 & 0.9 \\
$c$ & 0 & 0 & 0 & 0 \\
$d$ & 0 & 0 & 0 & 0 \\
$\mathscr{I}$ & $a$ & $b$ & $c$ & $d$ \\
\hline$a$ & 1 & 1 & 0.2 & 0.3 \\
$b$ & 1 & 1 & 0.7 & 0.1 \\
$c$ & 0.2 & 0.7 & 1 & 1 \\
$d$ & 0.3 & 0.1 & 1 & 1
\end{tabular}

\begin{tabular}{c|cccc}
$\mathscr{R}$ & $a$ & $b$ & $c$ & $d$ \\
\hline$a$ & 1 & 1 & 1 & 1 \\
$b$ & 1 & 1 & 1 & 1 \\
$c$ & 0.2 & 0.7 & 1 & 1 \\
$d$ & 0.3 & 0.1 & 1 & 1
\end{tabular}

Notice that $(\mathscr{P}, \mathscr{I})$ is a fuzzy total $T_{M}$-preorder. If we take $\alpha=0.75$ we have that

\begin{tabular}{c|cccc}
$\mathscr{R}_{\alpha}$ & $a$ & $b$ & $c$ & $d$ \\
\hline$a$ & 1 & 1 & 1 & 1 \\
$b$ & 1 & 1 & 1 & 1 \\
$c$ & 0 & 0 & 1 & 1 \\
$d$ & 0 & 0 & 1 & 1 \\
$\mathscr{P}_{\alpha}$ & $a$ & $b$ & $c$ & $d$ \\
\hline$a$ & 0 & 0 & 1 & 0 \\
$b$ & 0 & 0 & 0 & 1 \\
$c$ & 0 & 0 & 0 & 0 \\
$d$ & 0 & 0 & 0 & 0
\end{tabular}

Here $\mathscr{P}_{\alpha}(a, d)=0 \neq 1-\mathscr{R}_{\alpha}(d, a)=1-0=1$. 
Let $T$ be a $t$-norm and $S=T^{d}$ its dual $t$-conorm. We wonder if an additive fuzzy preference structure without incomparability $(\mathscr{P}, \mathscr{I})$, defined on a nonempty set $X$, is a fuzzy total $T$-preorder or not. When $T=T_{M}$ is the minimum t-norm, the answer is positive as next Proposition 9 shows.

Proposition 9. If an additive fuzzy preference structure without incomparability $(\mathscr{P}, \mathscr{I})$, defined on a nonempty set $X$, is representable, then it is a fuzzy total $T_{M}$-preorder. In particular, it is a fuzzy total $T$-preorder for every $t$-norm $T$.

Proof. This follows directly from Corollary 5 and Corollary 8.

Remark 7. The converse of Proposition 9 is not true, in general. As a matter of fact, there exist crisp total preorders that fail to be representable. Suitable examples are the lexicographic order on the real plane, or the linear order of the long line $27,25,28$.

Proposition 10. The concepts of representability and quasi-representability of additive fuzzy preference structures without incomparability, are not equivalent.

Proof. A glance at Example 2 shows that the additive fuzzy preference structure $(\mathscr{P}, \mathscr{I})$ introduced there is representable. To see this, observe that $(\mathscr{P}, \mathscr{I})$ is a fuzzy total $T_{M}$-preorder, so that $\mathscr{R}_{\alpha}$ is a (crisp) total preorder for every $\alpha \in[0,1]$, by Corollary 5. Since $X$ is finite, it is straightforward to see that each $\mathscr{R}_{\alpha}$ is representable ${ }^{25}$. Moreover, $(\mathscr{P}, \mathscr{I})$ is not quasi-representable because the relation $\mathscr{P}_{\alpha}$ fails to be negatively transitive when $\alpha=0.75$. (See also Remark 5).

Remark 8. As a matter of fact, if $X$ is a countable set and $(\mathscr{P}, \mathscr{I})$ is an additive fuzzy preference structure without incomparability defined on $X$, then the quasi-representability of $(\mathscr{P}, \mathscr{I})$ implies its representability. In other words, if we deal with countable sets, the concept of quasi-representability of additive fuzzy preference structures without incomparability is indeed more restrictive than the corresponding concept of representability. The proof of this fact will appear in next section. (See Theorem 12 below.)
The analogous of Proposition 9, assuming quasirepresentability instead of representability, is also true, as next Proposition 11 shows.

Proposition 11. If an additive fuzzy preference structure without incomparability $(\mathscr{P}, \mathscr{I})$, defined on a nonempty set $X$, is quasi-representable, then it is a fuzzy total $T_{M}$-preorder. In particular, it is a fuzzy total T-preorder for every t-norm $T$.

Proof. First we prove that $\mathscr{P}$ is $T_{M^{-}}$ asymmetric. If for every $a, b \in X$ we have that if $\min \{\mathscr{P}(a, b), \mathscr{P}(b, a)\}=\alpha \neq 0$, then $\mathscr{P}_{\alpha}(a, b)=$ $\mathscr{P}_{\alpha}(b, a)=1$. But this is a contradiction since, by hypothesis, $\mathscr{P}_{\alpha}$ is assumed to be asymmetric.

Let us prove now that $\mathscr{P}$ is $T_{M}$-negatively transitive, where $T_{M}$ stands for the minimum tnorm. To see this, take $a, b, c \in X$ and call $\alpha=\max \{\mathscr{P}(a, b), \mathscr{P}(b, c)\}$. Then $1-\alpha=$ $1-\max \{\mathscr{P}(a, b), \mathscr{P}(b, c)\}=\min \{1-\mathscr{P}(a, b), 1-$ $\mathscr{P}(b, c)\}$. Hence $(1-\mathscr{P})_{1-\alpha}(a, b)=(1-$ $\mathscr{P})_{1-\alpha}(b, c)=1$. This implies that $(1-$ $\mathscr{P})_{1-\alpha}(a, c)=1$, since $\mathscr{P}_{\alpha}$ is negatively transitive. Therefore $(1-\mathscr{P})(a, c) \geqslant 1-\alpha=\min \{1-$ $\mathscr{P}(a, b), 1-\mathscr{P}(b, c)\}$, so that $\mathscr{P}$ is $T_{M}$-negatively transitive.

By Corollary 7 we conclude that $(\mathscr{P}, \mathscr{I})$ is a fuzzy total $T_{M}$-preorder, so that by Corollary 8 it is also a fuzzy total $T$-preorder for any t-norm $T$.

\section{Representability of fuzzy total preorders}

A classical result by G. Cantor shows that every crisp total preorder $\precsim$ defined on a countable set $X$ is representable through a real-valued utility function $29,30,31,32,33,25$. (As a matter of fact, it can be proved that any total preorder on a countable set is isomorphic to the natural order on some suitable subset of the set $\mathbb{Q}$ of rational numbers: see Theorem 23 in the seminal book by Garret Birkhoff mentioned in the references ${ }^{33}$ ).

The generalization of Cantor's result to the fuzzy setting appears now as a direct consequence of previous facts. In particular, we recall that a necessary condition for the representability of an additive fuzzy preference structure without incomparability $(\mathscr{P}, \mathscr{I})$, defined on a nonempty set $X$, is that of 
$(\mathscr{P}, \mathscr{I})$ being a fuzzy total $T_{M}$-preorder, where $T_{M}$ denotes the minimum t-norm. (See Proposition 9). When the set $X$ is countable, that condition is also sufficient, as next Theorem 12 states.

Theorem 12. Let $X$ be a (nonempty) countable set. Let $(\mathscr{P}, \mathscr{I})$ be an additive fuzzy preference structure, without incomparability, defined on $X$. The following statements are equivalent:

(a) The structure $(\mathscr{P}, \mathscr{I})$ is a fuzzy total $T_{M^{-}}$ preorder.

(b) The structure $(\mathscr{P}, \mathscr{I})$ is representable.

Proof. The implication $(a) \Rightarrow(b)$ is, actually, a direct consequence of Cantor's classical theorem. The reason is that each $\alpha$-cut $\mathscr{R}_{\alpha}$ of the fuzzy weak preference relation $\mathscr{R}$ associated to $(\mathscr{P}, \mathscr{I})$ is a total preorder by Corollary 5. And, by Cantor's theorem, any total preorder on a countable set is representable through a real-valued utility function. Incidentally, it is indeed possible to represent the fuzzy preference structure $(\mathscr{P}, \mathscr{I})$ by means of a collection of rational-valued functions $\left\{u_{\alpha}: X \rightarrow \mathbb{Q}\right\}_{\alpha \in[0,1]}$ such that $a \mathscr{R}_{\alpha} b \Leftrightarrow u_{\alpha}(a) \leqslant u_{\alpha}(b)(a, b \in X)^{29,30,33}$.

The implication $(b) \Rightarrow(a)$ has been already stated in Proposition 9.

Remark 9. If $X$ is a nonempty countable set and $(\mathscr{P}, \mathscr{I})$ is an additive fuzzy preference structure without incomparability, defined on $X$, then by Remark 8 and Theorem 12 we have that if $(\mathscr{P}, \mathscr{I})$ is quasi-representable then $(\mathscr{P}, \mathscr{I})$ is representable. This is equivalent to say that $(\mathscr{P}, \mathscr{I})$ is a fuzzy total $T_{M}$-preorder. The converse of the first implication is not true, in general.

Let us provide now some characterization of the representability in the general case of a fuzzy total preorder defined on a nonempty set.

To do so, we first observe what happens in the crisp setting, where the question was solved by G. Debreu $^{31,32}$ among others. Thus, in the crisp approach, it is well-known that a condition that characterizes, in the general case (i.e.: the nonempty set $X$ may be uncountable), the representability through a real-valued utility function of a total preorder $\precsim$ defined on $X$, is the so-called perfect separability.
Namely, if $X$ is a nonempty set endowed with a total preorder $\precsim$, then $\precsim$ is representable through a real-valued utility function $u: X \rightarrow \mathbb{R}$ such that $a \precsim b \Leftrightarrow u(a) \leqslant u(b) \quad(a, b \in X)$ if and only if there exists a countable subset $D \subseteq X$ such that for every $a, b \in X$ with $a \prec b$ there exists $d \in D$ such that $a \precsim d \precsim b$. (See e.g. the first chapters of the book by D.S. Bridges and G.B. Mehta mentioned in the references) $)^{25}$.

Accordingly to the later result, we obtain a characterization of the representability of a fuzzy total $T_{M}$-preorder $(\mathscr{P}, \mathscr{I})$ defined on a nonempty set $X$. If $\mathscr{R}$ denotes the fuzzy weak preference relation associated to $(\mathscr{P}, \mathscr{I})$, then we need to express some condition that is equivalent to the representability of all the crisp total preorders $\mathscr{R}_{\alpha}$ induced by the $\alpha$ cuts of $\mathscr{R} \quad(\alpha \in[0,1])$. If we impose the perfect separability condition on each $\alpha$-cut $\mathscr{R}_{\alpha}$, we immediately obtain the desired result, as stated in the next Proposition 13.

Proposition 13. Let $X$ be a nonempty set and $(\mathscr{P}, \mathscr{I})$ a fuzzy total $T_{M}$-preorder defined on $X$. Let $\mathscr{R}$ denote its corresponding fuzzy weak preference relation. The fuzzy total preorder $(\mathscr{P}, \mathscr{I})$ is representable if and only if, for every $\alpha \in[0,1]$ there exists a countable subset $D_{\alpha} \subseteq X$ such that for each pair $(a, b) \in X \times X$ with $\mathscr{R}(b, a)<\alpha$ we can find an element $d_{\alpha}(a, b) \in D_{\alpha}$ such that $\alpha \leqslant$ $\min \left\{\mathscr{R}\left(a, d_{\alpha}(a, b)\right), \mathscr{R}\left(d_{\alpha}(a, b), b\right)\right\}$

Remark 10. Obviously, the sufficient condition introduced in Proposition 13 is, so-to-say, inelegant, because, as a matter of fact, it immediately splits as an uncountable collection of conditions (one for each $\alpha$-cut $\mathscr{R}_{\alpha}$ ). Unfortunately, and roughly speaking, this does not seem to be too operative in practice. In other words, the subset $\bigcup_{\alpha \in[0,1]} D_{\alpha}$ could be too big, even uncountable. Next Theorem 14 and Theorem 15 avoid this difficulty.

Theorem 14. Let $X$ be a nonempty set and $(\mathscr{P}, \mathscr{I})$ a fuzzy total $T_{M}$-preorder defined on X. The following assertions are equivalent:

(a) There exists a countable subset $D \subseteq X$ such that for each pair $(a, b) \in X \times X$ with $\mathscr{R}(b, a)<\alpha$ we can find an element $d(a, b) \in D$ such that $\alpha \leqslant \min \{\mathscr{R}(a, d(a, b)), \mathscr{R}(d(a, b), b)\}$. 
(b) The additive fuzzy preference structure, without incomparability, $(\mathscr{P}, \mathscr{I})$, defined on the nonempty set $X$, is representable.

Proof. The implication $(a) \Rightarrow(b)$ is a direct consequence of Proposition 13: just take $D_{\alpha}=D$ for every $\alpha \in[0,1]$.

Following Proposition 13, to prove the converse implication $(b) \Rightarrow(a)$, first we consider, for every $\alpha \in[0,1]$, a countable subset $D_{\alpha} \subseteq X$ such that for each pair $(a, b) \in X \times X$ with $\mathscr{R}(b, a)<\alpha$ we can find an element $d_{\alpha}(a, b) \in D_{\alpha}$ such that $\alpha \leqslant \min \left\{\mathscr{R}\left(a, d_{\alpha}(a, b)\right), \mathscr{R}\left(d_{\alpha}(a, b), b\right)\right\}$. Let $D=$ $\bigcup_{q \in \mathbb{Q} \cap[0,1]} D_{q}$. This subset $D$ is obviously countable, since $\mathbb{Q}$ is. Let us prove now that $D$ induces the perfect separability of all the $\alpha$-cuts $\mathscr{R}_{\alpha} \quad(\alpha \in$ $[0,1])$. To see this, let $a, b \in X$ be such that $\neg\left(b \mathscr{R}_{\alpha} a\right)$ holds. Equivalently, we have $\mathscr{R}(b, a)<$ $\alpha$. Fix a rational number $q \in[0,1] \cap \mathbb{Q}$ such that $\alpha \leqslant q$. Since $\mathscr{R}(b, a)<q$, by hypothesis there exists an element $d_{q}(a, b) \in D_{q} \subseteq D$ such that $q \leqslant$ $\min \left\{\mathscr{R}\left(a, d_{q}(a, b)\right), \mathscr{R}\left(d_{q}(a, b), b\right)\right\}$. Since $\alpha \leqslant q$, we get $\alpha \leqslant \min \left\{\mathscr{R}\left(a, d_{q}(a, b)\right), \mathscr{R}\left(d_{q}(a, b), b\right)\right\}$.

Coming back again to the statement of Proposition 13 , we may look at the crisp total preorders $\mathscr{R}_{\alpha}$ induced by the $\alpha$-cuts of $\mathscr{R}(\alpha \in[0,1])$ as a nested family of binary relations (in fact, total preorders) on $X$. Thus, if $\alpha \leqslant \beta$ then $\mathscr{R}_{\alpha}$ is coarser than $\mathscr{R}_{\beta}$ (i.e.: $\left.\mathscr{R}_{\beta} \subseteq \mathscr{R}_{\alpha}\right)$ and consequently $\left(\mathscr{R}_{\alpha}\right)^{c} \subseteq\left(\mathscr{R}_{\beta}\right)^{c}$, where $\mathscr{R}^{c}$ stands for the complement of a binary relation $\mathscr{R}$ defined on $X$. Having this in mind, we notice that the countable subset $D$ that meets condition (a) in Theorem 14 can actually be taken to be the set $D_{1}$ (see Proposition 13). Therefore $(\mathscr{P}, \mathscr{I})$ is representable if and only if $\mathscr{R}_{1}$ is. This fact obviously improves Proposition 13 and Theorem 14, as stated in the next Theorem 15.

Theorem 15. Let $X$ be a nonempty set and $(\mathscr{P}, \mathscr{I})$ a fuzzy total $T_{M}$-preorder defined on X. Then

(a) There exists a countable subset $D \subseteq X$ such that for every $a, b \in X$ such that $\mathscr{R}(b, a)<1$ it holds that $\min \{\mathscr{R}(a, d), \mathscr{R}(d, b)\}=1$ for some $d \in D \subseteq$ $X$.

(b) The fuzzy total $T_{M}$-preorder $(\mathscr{P}, \mathscr{I})$ is representable.
Proof. To prove the implication (a) $\Rightarrow$ (b), just observe that for each $\alpha$ and each $(a, b) \in X \times X$ such that $\mathscr{R}(b, a)<\alpha$, it holds that $\mathscr{R}(b, a)<\alpha \leqslant 1$, then $\mathscr{R}(b, a)<1$. By the hypothesis (a), there exists some $d \in D$ such that $\min \{\mathscr{R}(a, d), \mathscr{R}(d, b)\}=1$ in particular, $\min \{\mathscr{R}(a, d), \mathscr{R}(d, b)\} \geqslant \alpha$. This proves part (a) in the statement of Theorem 14 , so that $(\mathscr{P}, \mathscr{I})$ is representable.

The converse implication (b) $\Rightarrow$ (a) is a direct consequence of Theorem 14.

Example 3. Let $X=[0,1]$. Given $(x, y) \in X \times X$ define $\mathscr{P}(x, y)=y$ if $x<y$, and $\mathscr{P}(x, y)=0$ if $x \geqslant y$. Also, define $\mathscr{I}(x, y)=1-y$ if $x<y$, whereas $\mathscr{I}(x, y)=1-x$ if $y<x$, and finally $\mathscr{I}(x, x)=$ $1(x, y \in X=[0,1])$. Thus, the pair $(\mathscr{P}, \mathscr{I})$ becomes an additive fuzzy preference structure without incomparability, defined on $X=[0,1]$. The corresponding fuzzy weak preference relation $\mathscr{R}$ is given by $\mathscr{R}(x, y)=1-x$ if $y<x$ and $\mathscr{R}(x, y)=1$ if $x \leqslant y$. Given $\alpha \in[0,1]$ its $\alpha$-cut $\mathscr{R}_{\alpha}$ is given by $\mathscr{R}_{\alpha}(x, y)=1 \Leftrightarrow(x \leqslant y) \vee(y<x \leqslant 1-\alpha)$. It is straightforward to see that $(\mathscr{P}, \mathscr{I})$ is a fuzzy total $T_{M}$-preorder, so that by Corollary 5 , each $\mathscr{R}_{\alpha}$ is a (crisp) total preorder defined on $X$. For every $\alpha \in[0,1]$, take $D_{\alpha}=\mathbb{Q} \cap[0,1]$, which is obviously countable. (Observe that here all the $D_{\alpha}$ coincide). Given $x, y \in X$ such that $\mathscr{R}_{\alpha}(y, x)=0$, it follows that $x<y$ and $1-\alpha<y$ hold. We may find a rational number $d \in D$ such that $x<d<y$ and $1-\alpha<d$. Thus $\mathscr{R}_{\alpha}(y, d)=\mathscr{R}_{\alpha}(d, x)=0$. Therefore the fuzzy total $T_{M}$-preorder $(\mathscr{P}, \mathscr{I})$ is representable by Proposition 13.

\section{Further comments}

In the crisp case some characterizations of the representability of a total preorder have been stated in topological terms: for instance, if the order topology associated to $\precsim$ satisfy the second countability axiom, then $\precsim$ is representable, and viceversa. (For further details, see the first chapters in the book by D. S. Bridges and G. B. Mehta mentioned in the references $)^{25}$. Thus, it could also be interesting to look for topological conditions to characterize the representability of fuzzy total preorders. As far as 
we know this would constitute an open line for future research.

Another possible line for future research could be the consideration of techniques based on measure theory in order to get suitable numerical representations of additive fuzzy preference structures without incomparability, and, in particular of fuzzy total $T$-preorders with respect to some t-norm $T$. In the crisp case, some studies on the representability of certain kinds of orderings, in terms of measure theory, have already been considered in the specialized literature $34,35,36,25,22$.

\section{Acknowledgments}

This work has been supported by the research projects MTM2009-12872-C02-02 and MTM201017844 (Spain).

Thanks are given to the editors Luis Martínez and Humberto Bustince, as well as to two anonymous referees, for their valuable suggestions and comentd.

\section{References}

1. I. Montes, S. Díaz and S. Montes, On complete fuzzy preorders and their characterizations, Soft Computing 15, 1999-2011 (2010).

2. S. Díaz, B. De Baets and S. Montes, On the Ferrers property of valued interval orders, TOP 19 (2), 421427 (2011).

3. S. Díaz, E. Induráin, B. De Baets and S. Montes, Fuzzy semi-orders: the case of $t$-norms with zero divisors, Fuzzy Sets and Systems 184, 52-67 (2011).

4. E. Induráin, D. Martinetti, S. Montes, S. Díaz and F. J. Abrísqueta, On the preservation of semiorders from the fuzzy to the crisp setting, Internat. J. Uncertain. Fuzziness Knowledge-Based Systems 19 (6), 899-920 (2011).

5. M. Roubens and Ph. Vincke, Preference Modelling, Springer-Verlag, Berlin, 1985.

6. J. Fodor, M. Roubens, Fuzzy Preference Modelling and Multicriteria Decision Support. Kluwer Academic Publishers, Dordrecht, 1994.

7. E. P. Klement, R. Mesiar, E. Pap, Triangular Norms, Kluwer Academic Publishers, 2000.

8. B. De Baets and J. Fodor, Twenty years of fuzzy preference structures (1978-1997), Belg. J. Oper. Res. Statist. Comput. Sci. 37, 61-82 (1997).
9. B. Van De Walle, B. De Baets and E. Kerre, Characterizable fuzzy preference structures, Ann. Oper. Res. 80, 105-136 (1998).

10. H. Nurmi, Approaches to collective decision making with fuzzy preference relations, Fuzzy Sets and Systems 6, 249-259 (1981).

11. B. Dutta, Fuzzy preferences and social choice, Math. Social Sci. 13, 215-229 (1987).

12. C. R. Barret, P. Pattanaik and M. Salles, Rationality and aggregation of preferences in a fuzzy framework, Fuzzy Sets and Systems 49, 9-13 (1992).

13. A. Billot, An existence theorem for fuzzy utility functions: A new elementary proof, Fuzzy Sets and Systems 74, 271-276 (1995).

14. G. Richardson, The structure of fuzzy preferences, Soc. Choice Welf. 15, 359-369 (1998).

15. J. L. García-Lapresta and B. Llamazares, Aggregation of fuzzy preferences: some rules of the mean, Soc. Choice Welf. 17, 673-690 (2000).

16. S. Ovchivnnikov, Numerical representation of fuzzy transitive relations, Fuzzy Sets and Systems 126, 225232 (2000).

17. U. Bodenhofer, B. De Baets and J. Fodor, A compendium of fuzzy weak orders: representations and constructions, Fuzzy Sets and Systems 158, 811-829 (2007).

18. L. A. Fono and N. G. Andjiga, Utility function of fuzzy preferences on a countable set under max-star transitivity, Soc. Choice Welf. 28, 667-683 (2007).

19. L. A. Fono and M. Salles, Continuity of utility functions representing fuzzy preferences, Soc. Choice Welf. 37, 669-682 (2011).

20. M. J. Campión J. C. Candeal, R. G. Catalán, J. R. De Miguel, E. Induráin and J. A. Molina, Aggregation of preferences in crisp and fuzzy settings: functional equations leading to possibility results, Internat. J. Uncertain. Fuzziness Knowledge-Based Systems 19 (1), 89-114 (2011).

21. S. Díaz, B. De Baets and S. Montes, Transitivity and negative transitivity in the fuzzy setting, Advances in Intelligent and Soft Computing 117, 91-100 (2012).

22. G. Bosi, J. C. Candeal, E. Induráin, E. Olóriz and M. Zudaire, Numerical representations of interval orders, Order 18, 171-190 (2001).

23. M. J. Campión, J. C. Candeal and E. Induráin, Representability of binary relations through fuzzy numbers, Fuzzy Sets and Systems 157, 1-19 (2006).

24. D. Scott and P. Suppes, Foundational aspects of theories of measurement, J. Symbolic Logic 23, 113-128 (1958).

25. D. S. Bridges and G.B. Mehta, Representations of Preference Orderings, Springer, Berlin, 1995.

26. J. C. Candeal and E. Induráin, Lexicographic behaviour of chains, Arch. Math. (Basel) 72, 145-152 (1999). 
27. L. A. Steen and J. A. Seebach Jr., Counterexamples in Topology, Holt, Rinehart and Winston, New York, 1970.

28. J. C. Candeal, J. R. De Miguel, E. Induráin and G.B. Mehta, Utility and entropy, Econom. Theory 17, 233238 (2001).

29. G. Cantor, Beiträge zur Begründung der transfinite Mengenlehre I, Math. Ann. 46, 481-512 (1895).

30. G. Cantor, Beiträge zur Begründung der transfinite Mengenlehre II, Math. Ann. 49, 207-246 (1897).

31. G. Debreu, Representations of a preference ordering by a numerical function, in Thrall, R., Coombs, C. and Davies, R. (eds.) Decision processes, New York, John Wiley, 1954.
32. G. Debreu, Continuity properties of Paretian utility, Internat. Econom. Rev. 5, 285-293 (1964).

33. G. Birkhoff, Lattice Theory. (Third edition), American Mathematical Society, Providence RI, 1967.

34. J. C. Candeal-Haro and E. Induráin-Eraso, Utility functions for partially ordered topological groups, Proc. Amer. Math. Soc. 115, 765-767 (1992).

35. J. C. Candeal-Haro and E. Induráin-Eraso, Utility representations from the concept of measure, Math. Social Sci. 26, 51-62 (1993).

36. J. C. Candeal-Haro and E. Induráin-Eraso, Utility representations from the concept of measure: a corrigendum, Math. Social Sci. 28, 67-69 (1994). 